\title{
Les dépôts en couches minces par ablation laser en régime d'impulsions ultra-courtes : état de l'art et perspectives
}

\author{
E. Fogarassy \\ CNRS-PHASE, BP. 20, 67037 Strasbourg cedex 2, France
}

\begin{abstract}
Résumé : Le développement récent de sources femtosecondes de très fortes intensités $\left(10^{14}-10^{15} \mathrm{~W} / \mathrm{cm}^{2}\right)$ ouvre des perspectives nouvelles au procédé de dépôt en couches minces par photo-ablation, en raison notamment, du caractère athermique de l'interaction laser-matière en impulsions ultra-courtes, et de la génération de plasmas très énergétiques. Après avoir rappelé les mécanismes fondamentaux mis en jeu dans ce régime et les conséquences attendues sur les propriétés des dépôts, nous ferons un bilan des travaux réalisés dans ce domaine, qui concernent très majoritairement les films de carbone. Nous tenterons aussi de dégager les perspectives de cette technique, liées en partie au développement futur des sources femtosecondes.
\end{abstract}

\section{INTRODUCTION}

Les dépôts en couches minces par ablation laser ( Pulsed Laser Deposition : PLD) font l'objet de recherches extrèmement actives depuis la découverte des composés supraconducteurs à haute température critique, à la fin des années 80 [1]. C'est, en effet, à cette date que furent réalisées par PLD les premières couches épitaxiées de ces oxydes complexes. Depuis, le succés rencontré par cette technique est en partie lié au fait qu'elle représente, pour certains matériaux d'importance technologique, une alternative très prometteuse aux méthodes classiques de dépôt par voies physiques (PVD) ou chimiques (CVD). La très grande majorité des travaux menés à ce jour utilise des lasers ultra-violets capables de fournir, en régime nanoseconde, des intensités comprises entre $10^{8}$ et $10^{11} \mathrm{~W} / \mathrm{cm}^{2}$. Cependant, l'arrivée récente sur le marché [2] de sources femtosecondes de fortes intensités $\left(10^{14}\right.$ à $\left.10^{15} \mathrm{~W} / \mathrm{cm}^{2}\right)$ ouvre des perspectives nouvelles au procédé de dépôt par photoablation, en raison notamment, du caractère athermique de l'interaction laser-matière en impulsions ultra-courtes, et de la génération de plasmas très énergétiques. Après avoir rappelé les mécanismes fondamentaux mis en jeu dans ce régime et les conséquences attendues sur les propriétés des dépôts, nous ferons un bilan des travaux réalisés dans ce domaine, qui concernent très majoritairement les films de carbone. Nous tenterons aussi de dégager les perspectives de cette technique, liées en partie au développement futur de sources femtosecondes industrielles de plus en plus fiables et performantes.

\section{MECANISMES DE BASE DE LA PLD EN IMPULSIONS ULTRA-COURTES}

Les propriétés des dépôts PLD dépendent des processus complexes mis en jeu aux différentes étapes du procédé. Ils concernent, d'une part, l'interaction d'un faisceau laser intense avec une cible massive et les mécanismes de formation d'un plasma luminescent, d'autre part, l'expansion adiabatique de ce plasma, enfin la formation d'un dépôt résultant de la collecte des espèces émises. Nous allons maintenant passer en revue les différentes étapes de ce processus et analyser ses spécificités en régime d'impulsions ultra-courtes. 


\subsection{Interaction laser-matière et formation du plasma}

L'analyse du processus d'ablation repose sur une bonne connaissance des mécanismes de couplage entre un faisceau laser incident et la surface du solide irradié. Ces mécanismes sont très complexes puisqu'ils dépendent, à la fois des caractéristiques de la source laser ( fluence, longueur d'onde, profil spatio-temporel de l'impulsion, taux de répétition) et des propriétés optiques et thermiques du matériau considéré, qui peuvent évoluer considérablement au cours de l'irradiation à cause des effets thermiques, de phénomènes de transitions de phase, ou encore de réactions chimiques. Quelque soit la nature du matériau considéré (diélectrique, semiconducteur, métal), les études récentes [3-7] montrent le rôle particulièrement important joué par la durée de l'impulsion laser sur le phénomène d'ablation, pour trois raisons : (i) Les intensités extrèmement élevées émises par les sources à impulsions ultra-courtes $\left(\mathrm{I} \sim 10^{14}\right.$ $10^{15} \mathrm{~W} / \mathrm{cm}^{2}$ ), favorisent les processus d'absorption multiphotoniques. (ii) En régime femtoseconde, l'impulsion laser n'interagit plus avec le plasma qui se forme après l'extinction de la source d'excitation. Dans ces conditions, le taux d'espèces ablatées devient indépendant de la dimension de l'impact laser (iii) En régime femtoseconde, la profondeur de la zone affectée thermiquement devient négligeable. En effet, la longueur de diffusion thermique $\left(\mathrm{L}_{\text {th }}\right.$ $\sim(D . \tau)^{1 / 2}$ avec D : diffusivité thermique et $\tau$ : durée d'impulsion ) dans le matériau diminue avec la durée de l'impulsion laser, ce qui conduit à une réduction des seuils d'ablation en impulsions courtes. L'autre conséquence importante porte sur la nature même du processus d'ablation: Pour des impulsions qui excèdent la centaine de picosecondes ( $\tau>100 \mathrm{ps}$ ), le phénomène est assisté thermiquement : la zone irradiée, portée à haute température, atteint son point de fusion puis d'évaporation. Dans ce régime, le processus de formation du plasma est initié à partir d'une phase liquide. A l'inverse, lorsque la durée d'impulsion diminue ( $\tau<$ $10 \mathrm{ps}$ ), le dépôt de l'énergie photonique à la surface de l'échantillon, en un temps extrêmement bref, conduit à un processus de transition directe solide-vapeur (ou solideplasma), sans intervention d'une phase liquide. Dans ce régime, le phénomène d'ablation résulte principalement de processus d'ionisation multi-photoniques.

\subsection{Expansion adiabatique du plasma}

Au terme de l'impulsion laser, le plasma est soumis à une détente adiabatique. L'équation de Dawson [8] donne accès à l'évolution spatio-temporelle du plasma sous vide :

$X(t) \cdot d^{2} X(t) / d t^{2}=Y(t) \cdot d^{2} Y(t) / d t^{2}=Z(t) \cdot d^{2} Z(t) / d t^{2}=K \cdot T_{0} / M \cdot\left(X_{0} \cdot Y_{0} \cdot Z_{0} / X(t) \cdot Y(t) \cdot Z(t)\right)^{\gamma-1}$

$X_{0}, Y_{0}, Z_{0}$ et $X(t), Y(t), Z(t)$ représentent les coordonnées orthogonales du front du plasma, à l'instant initial (au terme de l'impulsion laser), et à l'instant t. Le plasma quasi-isotherme est caractérisé par une température $T_{0}$, à $t=0$. Enfin, $\gamma=C_{p} / C_{v}$ correspond au rapport des chaleurs spécifiques à pression et à volume constant.

Les dimensions initiales du plasma dans le plan de l'échantillon ( $\mathrm{Y}_{0}$ et $\left.\mathrm{Z}_{0}\right)$, comparables à celles de l'impact laser, sont de un à deux ordres de grandeurs plus élevées que $\mathrm{X}_{0}$. En conséquence, L'équation (1) montre que l'accélération initiale des espèces du plasma dans la direction $\mathrm{X}$, normale à la cible, est très supérieure à l'accélération dans le plan transverse. Ceci explique que le distribution angulaire de la matière ablatée soit systématiquement piquée dans cette direction. En diminuant la durée d'impulsion, on tend à réduire la dimension initiale $\mathrm{X}_{0}$ du plasma, ce qui a pour principale conséquence d'augmenter l'accélération initiale des espèces ablatées perpendiculairement à la surface de la cible, et de produire des plasmas très énergétiques, constitués d'espèces dont l'énergie cinétique peut atteindre plusieurs milliers d'eV. (Tableau II). Notons que des modifications de nature chimique (nature et 
proportion des différentes espèces : ions, molécules, agrégats) de ces plasmas ont aussi été mis en évidence, qui ne sont pas sans conséquences sur les propriétés des dépôts PLD.

\subsection{Dépôts en couches minces}

Les modifications attendues des propriétés des films élaborés en régime femtoseconde, sont de differentes natures. Elles résultent, tout d'abord, du caractère athermique de l'interaction laser-matière dans ce régime. En effet, celui-çi devrait favoriser le transfert quasi-congruent des espèces ablatées entre la cible et le substrat, et la conservation des compositions lors du dépôt de certains matériaux complexes (ex: polymères). Par ailleurs, l'absence (ou la réduction) de la zone fondue lors du processus d'ablation devrait éliminer (ou diminuer) le phénomène d'émission de gouttelettes liquides, responsable de la dégradation des propriétés de surface des dépôts [9]. Les caractéristiques physico-chimiques des plasmas produits en régime femtoseconde : Taux d'ionisation, énergie cinétique et masse des espèces ablatées, devraient aussi influer sur le processus de croissance et la structure des films, comme le confirment les résultats des expériences réalisées sur le carbone [13]. Par ailleurs, les vitesses de dépôt instantanées extrêmement élevées atteintes en régime femtoseconde, pourraient avoir des conséquences sur les propriétés des couches en croissance [22]. Enfin, l'expansion de ces plasmas très énergétiques et fortement ionisés, dans un environnement controlé (gaz neutre ou réactif) devrait favoriser les processus réactifs en phase gazeuse et conduire à la synthèse de nouveaux matériaux ou de nouvelles structures.

\section{ETAT DE L'ART. L'EXEMPLE DU CARBONE}

Le tableau I résume l'ensemble des publications relatives aux dépôts en couches minces par ablation laser en impulsions courtes. A l'exception des premières expériences menées en France, au début des années 90 , avec des sources picosecondes, ces travaux ont été réalisés au moyen de sources femtosecondes émettant dans le visible (Ti : Saphir, colorant) ou l'ultraviolet (Excimère). Bien que des recherches exploratoires aient été menées très récemment, par ablation laser réactive, sur la croissance de films de nitrures $[18,20]$ et d'oxydes $[21,22]$, l'essentiel du travail a porté sur le carbone amorphe, type DLC (Diamond-Like-Carbon), dont il est intéressant d'analyser plus en détails les résultats. Le tableau II présente une comparaison de quelques propriétés caractéristiques de films DLC, préparés dans les deux régimes d'ablation: nanoseconde [23] et femtoseconde [15]. Le pourcentage de liaisons $\mathrm{sp}^{3}$ dans la couche de carbone, et sa largeur de bande interdite $\left(E_{G}\right)$, atteignent une valeur maximale en régime nanoseconde, pour des énergies cinétiques $(\mathrm{Ec})$ des ions carbone dans le plasma de l'ordre de $90 \mathrm{eV}$. En impulsion ultra-courte, on observe une dégradation notable de ces propriétés, principalement due à l'évolution des caractéristiques énergétiques du plasma laser. En effet, l'énergie cinétique très élevée $(\mathrm{Ec}>1000 \mathrm{eV})$ des ions $\mathrm{C}^{+}$ablatés en régime femtoseconde, peut avoir des conséquences néfastes sur les propriétés structurelles et morphologiques des films en croissance. L'état de surface des dépôts préparés dans les deux régimes d'ablation est de très bonne qualité (peu ou pas de gouttelettes). Cependant, on note en régime femtoseconde à forte intensité ( $\mathrm{I}>1 \times 10^{15} \mathrm{~W} / \mathrm{cm}^{2}$ ), une augmentation de la rugosité de surface, due à la présence de particules de taille micronique, dont l'origine n'est pas clairement établie. 
Tableau I

\begin{tabular}{|c|c|c|}
\hline AUTEUR & MATERIAU & LASER \\
\hline - W. MARINE (1989) & YbaCuO & Nd:YAG $(1064 \mathrm{~nm}, 35 \mathrm{ps})$ \\
\hline - W. MARINE (1990) & Silicium & Nd:YAG (1064 nm, 35 ps) \\
\hline - D. GIVORD (1990) & Intermétalliques & Nd :YAG (355 nm, $35 \mathrm{ps})$ \\
\hline - F. MULLER et K. MANN (1993) [13] & Carbone & Excimère $(248 \mathrm{~nm}, 500 \mathrm{fs})$ \\
\hline - R.K. SINGH et P.P. PRONKO (1995) [14] & Carbone & $\mathrm{Ti}$ : Saphir $(780 \mathrm{~nm}, 500 \mathrm{fs})$ \\
\hline - R.K. SINGH et P.P. PRONKO (1999) [15] & Carbone & $\mathrm{Ti}:$ Saphir $(780 \mathrm{~nm}, 100 \mathrm{fs})$ \\
\hline - P.S. BANKS (1999) & Carbone & Ti: Saphir $(780 \mathrm{~nm}, 120 \mathrm{fs})$ \\
\hline - M. OKOSHI (1999) & Carbone & $\mathrm{Ti}:$ Saphir $(780 \mathrm{~nm}, 130 \mathrm{fs})$ \\
\hline - T. SZORENYI et E. FOGARASSY (1999) [18] & $\mathrm{CN}_{\mathrm{x}}$ & Excimère $(248 \mathrm{~nm}, 500 \mathrm{fs})$ \\
\hline - F. GARRELIE et P. LAPORTE (2000) [19] & Carbone & Ti : Saphir $(780 \mathrm{~nm}, 150 \mathrm{fs})$ \\
\hline - Z. ZHANG et P.P. PRONKO (1999) & TiN, BN & $\mathrm{Ti}:$ Saphir $(780 \mathrm{~nm}, 200 \mathrm{fs})$ \\
\hline - Z. ZHANG et P.P. PRONKO (2000) & $\mathrm{SnO}_{2}$ & $\mathrm{Ti}$ : Saphir (780 nm, $80 \mathrm{fs})$ \\
\hline - J. PERRIERE, E. MILLON, D. HULIN (2001) [22] & $\mathrm{ZnO}$ & Colorant $(620 \mathrm{am}, 90 \mathrm{fs})$ \\
\hline
\end{tabular}

Tableau II

\begin{tabular}{|c|c|c|c|c|c|c|}
\hline Source & $\mathrm{I}\left(\mathrm{W} / \mathrm{cm}^{2}\right)$ & $\begin{array}{c}\text { Cible } \\
\text { (sous vide) }\end{array}$ & $E_{C}(e V)$ & $\begin{array}{c}\% \text { de sp }{ }^{3} \\
(\max .)\end{array}$ & $\begin{array}{c}\mathrm{E}_{\mathrm{j}}(\mathrm{eV}) \\
\text { (max.) }\end{array}$ & Etat de surface \\
\hline $\begin{array}{l}\text { Excimère[23] } \\
\text { (ArF, } 25 \text { ns.) }\end{array}$ & $\begin{array}{l}3 \times 10^{7} \\
5 \times 10^{8}\end{array}$ & Graphite & $40-100$ & $\begin{array}{c}75 \\
(\mathrm{Ec}=90 \mathrm{eV})\end{array}$ & $\begin{array}{c}2.1 \\
\left(E_{c}=90 \mathrm{eV}\right)\end{array}$ & Très bon \\
\hline $\begin{array}{l}\text { Ti:Saphir[15] } \\
\text { (100 fs.) }\end{array}$ & $\begin{array}{l}3 \times 10^{14} \\
6 \times 10^{15}\end{array}$ & Graphite & $300-2000$ & $\begin{array}{c}60 \\
(E c=300 \mathrm{eV})\end{array}$ & $\begin{array}{c}1.4 \\
(E c=300 \mathrm{eV})\end{array}$ & $\begin{array}{c}\text { Dégradation } \\
\left(\mathrm{I}>1 \times 10^{15} \mathrm{~W} / \mathrm{cm}^{2}\right)\end{array}$ \\
\hline
\end{tabular}

\section{PERSPECTIVES}

Les résultats obtenus à ce jour en PLD femtoseconde, posent probablement plus de questions qu'elles n'en résolvent. L'optimisation du procédé reposera, tout d'abord, sur une meilleure connaissance des mécanismes fondamentaux de l'ablation laser en impulsions ultra-courtes. Au niveau expérimental, la priorité devrait être donnée à l'élaboration de matériaux en 
couches minces difficilement réalisables en régime nanoseconde, tels que les composés organiques (polymères, bio-matériaux ...). Par ailleurs, il s'agira, pour des raisons technologiques évidentes, de mettre à profit la possibilité d'éliminer ou de réduire l'émission des gouttelẹttes en régime femtoseconde, afin d'améliorer l'état de surface des films PLD. La spécificité des plasmas femtosecondes pourrait aussi favoriser la synthèse de nouveaux matériaux par ablation laser en atmosphère controlée (gaz neutre ou réactif) et la formation (à haute pression) de nanostructures. Enfin, il est important de rappeler que ces perspectives sont étroitement liées aux performances actuelles et futures des sources femtosecondes, dont il s'agira de bien maitriser les paramètres clés: Intensité, durée, profil spatio-temporel des impulsions et longueur d'onde d'émission ( lasers UV à impulsions ultra-courtes).

\section{Références}

1. Voir par exemple «Pulsed Laser Deposition of Thin Films » édité par D.B. Chrisey et G.K. Hubler ( Wiley-Interscience, New-York, 1994).

2. F. Savatier, l'Usine Nouvelle $n^{\circ} 2696$ ( 1999) 56.

3. S. Preuss, E. Matthias, M. Stuke, Appl. Phys.A 59 (1994) 79.

4. W. Kautek, J. Kruger, SPIE Proc.Vol. 2207 (1994) 600.

5. B.N. Chichkov, C. Momma, S. Nolte, F. von Alvensleben, A. Tunnermann, Appl. Phys.A $63(1996) 109$.

6. B.C. Stuart, M.D. Feit, S. Herman, A.M. Rubenchick, B.W. Shore, M.D. Perry, Phys. Rev. B 53, n²4 (1996) 1749.

7. X. Liu, D. Du, G. Mourou, IEEE J. of Quantum Electron. 33, n¹0 (1997) 1706.

8. J. Dawson, P. Kaw, B. Green, Phys. Fluids 12 (1969) 875.

9. C. Fuchs, E. Fogarassy, MRS Proc. Vol.169 (1990) 517.

10. W. Marine, M. Peray, Y. Mathey, D. Pailharey, Appl. Surf. Sci. 43 (1989) 377.

11. W. Marine, J.M. Scotto d'Aniello, J. Marfaing, Appl. Surf. Sci. 46 (1990) 239.

12. J.P. Gavigan, D. Givord, A. Lienard, O.F.K. Mc Grath, J.P. Rebouillat, Y. Souche, MRS Proc. Vol.191 (1990) 49.

13. F. Muller, K. Mann, P. Simon, J.S. Bernstein, G.J. Zaal, SPIE Proc. Vol. 1858 (1993) 464.

14. F. Qian, R.K. Singh, S.K. Dutta, P.P. Pronko, Appl. Phys. Lett. 67 (1995) 3120.

15. F. Qian, V. Craciun, R.K. Singh, S.K. Dutta, P.P. Pronko, J. Appl. Phys. 86 (1999) 2281.

16. P.S. Banks, L. Dinh, B.C. Stuart, M.D. Feit, A.M. Komashko, A.M. Rubenchik, M.D. Perry, W. Mc Lean, Appl. Phys. A 69 [Suppl.] (1999) S347.

17. M. Okoshi, S. Higuchi, M. Hanabusa, J. Appl. Phys. 86 (1999) 1768.

18. T. Szorenyi, E. Fogarassy, C. Fuchs, J. Hommet, F. Le Normand, Appl. Phys. A 69 [Suppl.] (1999) S941.

19. F. Garrelie, C. Jonin, A. Catherinot, C. Champeaux, E. Baubeau, A. Audouard, P. Laporte, UVX 2000, Ile de Porquerolles (16-19 Mai 2000).

20. Z. Zang, P.A. Van Rompay, J.A. Nees, R. Clarke, X. Pan, P.P. Pronko, Appl. Surf. Sc. (1999) 727.

21. Z. Zang, P.A. Van Rompay, J.A. Nees, C.A. Stewart, X.Q. Pan, L. Fu, P.P. Pronko, SPIE Proc. Vol.3935 (2000).

22. E. Millon, O. Albert, J.C. Loulergue, J. Etchepare, D. Hulin, W. Seiler, J. Perrière, A paraître dans J. of Appl. Phys. (2001)

23. D.H. Lowndes, V.I. Merkulov, A.A. Puretzky, D.B. Geohegan, G.E. Jellison Jr., C.M. Rouleau, T. Thundat, MRS Proc. Vol. 526 (1998) 325. 